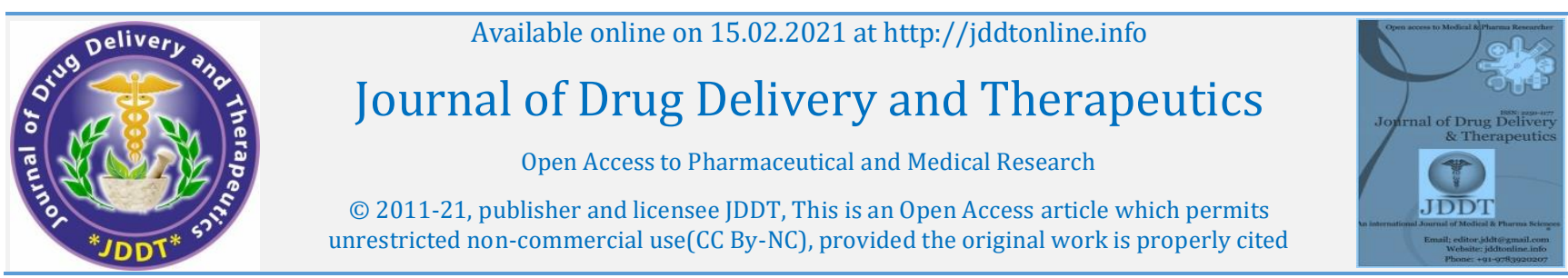

\title{
Pattern of antibiotics resistance and phenotypic characterization of Multidrug resistant bacteria isolates in four hospitals of Littoral region, Cameroon
}

\author{
Takemegni Wandji Jonas Merlin ${ }^{1,2}$, Assob Nguedia Jules Clement ${ }^{1,7}$, Ateudjieu Jérôme ${ }^{3,4,5}$, Enow Orock George ${ }^{1}$, \\ Mbopi-Keou Francois-Xavier ${ }^{8}$, Ngowe Ngowe Marcelin 6,7
}

\author{
1. Department of Medical Laboratory Sciences, Faculty of Health Sciences, University of Buea, Buea, Cameroon \\ 2. Department of Medical Laboratory Sciences, New-Bell District Hospital, Douala, Cameroon \\ 3. Division of Health Research, M. A. SANTE (Meilleur Accès aux Soins de Santé/Better Access to Health Care), Yaoundé, Cameroon \\ 4. Department of Public Health, University of Dschang, Dschang, Cameroon \\ 5. Division of Health Operations Research, Ministry of Public Health, Yaoundé, Cameroon \\ 6. Department of Surgical Sciences, Faculty of Medicine and pharmaceutical Sciences, University of Douala, Douala, Cameroon \\ 7. Department of Biological Sciences, Faculty of Medicine and pharmaceutical Sciences, University of Douala, Douala, Cameroon \\ 8. Department of Microbiology, Virology and Immunology, Faculty of Medicine and Biomedical Sciences, University of Yaoundé I, Yaoundé, Cameroon
}

\section{Article Info: \\ Article History: Received 10 Dec 2020; Review Completed 16 Jan 2021 \\ Accepted 24 Jan 2021; \\ Available online $15 \mathrm{Feb} 2021$ \\ Cite this article as:

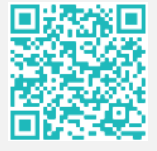 \\ Takemegni Wandji JM, Assob Nguedia JC, Ateudjieu J, Enow OG, Mbopi-Keou F-X, Ngowe NM, Pattern of antibiotics resistance and phenotypic characterization of Multidrug resistant bacteria isolates in four hospitals of Littoral region, Cameroon, Journal of Drug Delivery and Therapeutics. 2021; 11(1-s):20-30 DOI: http://dx.doi.org/10.22270/jddt.v11i1-s.4700 \\ *Address for Correspondence: \\ Takemegni Wandji Jonas Merlin, Department of Medical Laboratory Sciences, Faculty of Health Sciences, University of Buea, P.O Box 63, Buea SW Region Cameroon}

\section{Abstract}

Objective: To describe the pattern of antibiotics resistance and phenotypic characterization of Multidrug resistant bacteria isolates in four hospitals of Littoral region, Cameroon.

Methods: We conducted a descriptive hospital based cross-sectional study from December 2018 to May 2019. A simple random sampling was used to swap 10 selected equipment and 10 materials in the mornings after disinfection but before the start of work in seven units. After inoculation in four agar media consecutively (Eosine Metyleine blue, Cled, Manitol salt agar and blood agar ) and incubated in appropriate conditions, the Kirby-Bauer disk-diffusion method was used for antimicrobial susceptibility test.

Results: Among 50.4\% (119/236) showed positive bacteria growth, a total of 89 (13 species), predominant bacteria and those more likely to cause nosocomial infections were selected and tested each one to 18 antibiotics. There was high level of resistance to Penicillin (amoxicillin (77.5\%) and Oxacillin (76.4\%)), followed by $3 G$ Cephalosporine (Ceftazidime (74.2\%)) and Monobactam (Aztreonam (70.8\%)). Although the least level of resistance was observed in Carbapenem (imipenem (5.6\%)). The overall prevalence of MDRB was 62.9\% (56/89). MRSA were the mostly detected $57.5 \%$ (30/89), followed by ESBL 10.1\% (9/89). Military hospital of Douala and Emergency unit was the MDRBs dominantly contaminated area respectively $39.3 \%(22 / 56)$ and $17.9 \%(10 / 56)$.

Conclusion: MDRB occurred to be a current public health problem as well as hospital surfaces are worrying reservoir that can be spread to patient, health professionals and visitors.

Keywords: Antibiotic resistance, susceptibility test, multidrug-resistant bacteria, Hospital facilities, Units, Littoral Region-Cameroon

\section{INTRODUCTION}

Contaminated hospital environment has been recognized to be the most significant reservoir of bacteria causing nosocomial infections ${ }^{1-4}$. Nosocomial infections (NI) known as Health-care Associated Infections (HAIs) and also Hospital-Acquired Infections, are infections in a hospitalized patient not present or incubating on admission. It occurs after 48 hours or more of hospital admission or within 30 days after discharge ${ }^{5-7}$. These pathogens, present in air, hands and surface including equipment colonize patients, staff and visitors by dynamic transmission (to, from and between them $)^{8}, 9$. Several lines of evidence suggest that Nosocomial infections and resistant organisms as well as multidrug resistant bacteria (MDRB) often increase each other ${ }^{10,11}$. Thus, many compelling observations abound in the same direction that include: Antimicrobial resistance is more prevalent in nosocomial bacterial strains than in those from community-acquired, during outbreaks of nosocomial infection, patients infected with resistant strains are more likely than control patients to have received prior antimicrobials, increasing length of hospital stay (LOS) of NI patient to hospital increases the likelihood of colonization with resistant organism, a quarter of HAIs in long-term acute care settings are caused by antibiotic-resistant bacteria and HAIs mostly caused by multi drug resistant (MDR) organisms ${ }^{12-15}$.

These organisms are those with acquired poor susceptibility to one, two at list three or more antimicrobial categories 16 , 17. Antibiotic resistance is one of the biggest public health challenges of our time. The World Health Organization (WHO) considers Antimicrobial Resistance (AMR) to be one of the greatest threats to human health in the 21st Century. It is estimated that by 2050 if the AMR threat was not properly tackled, it would lead to 10 million people dying every year and a reduction of $2-3.5 \%$ in Gross Domestic Product (GDP) with an overall cost of 100 trillion USD ${ }^{18,} 19$. Each year in the U.S., at least 2.8 million people get an antibiotic-resistant infection, and more than 35,000 people $\mathrm{die}^{20}$. This burden is expected to be relatively higher in sub- 
Saharan Africa with a drop in GDP of US \$2895billion, representing $20 \%$ of the region's total economic output 21 . Furthermore, AMR remains a significant One Health problem, affecting humans, animals, and the environment ${ }^{20}$. Hence it is accessed that the selection of resistant organisms somewhere, worldwide, even within an animal may have long-term implication for vegetable and human health globally22, 23. In addition, the development of such infections causes patient's discomfort, delayed healing, anxiety, longer stays at hospitals and adds to cost of healthcare services significantly ${ }^{24}$. Dedicated prevention and infection control efforts worldwide are working to reduce the number of infections and deaths caused by antibiotic-resistant germs, but the number of people facing antibiotic resistance is still too high. In Cameroon despite the elaboration of national guidelines and directives on the prevention of nosocomial infections associated with surveillance and fight recommendations ${ }^{25}$, as CDC reported in 2019 in their antimicrobial resistance treat, preventing the spread of germs from the healthcare environment, incomplete adoption of the containment strategy, inconsistent implementation of some CDC recommendations, continued vigilance against serious threats like MDR remain challenges in healthcare facilities ${ }^{20}$. In addition, a study in Cameroon in 10 health facilities to five districts of Yaoundé with the aim to assess their compliance levels with recommendations on national NIs control programs, showed in the item of "infection outbreaks and surveillance of NIs" that all the health facilities had a score below $15 \%$ and based on previous research, demonstrated the need and follow-up of epidemiological surveillance of new pathogens and their antibiotic resistance ${ }^{8}$. More action is needed to fully protect people. While AMR cause substantial morbidity and mortality, the availability of timely and accurate epidemiological information in various hospitals surface reservoir is one of the essential approach to fight this priority public health problem. Several studies on AMR have been published in Cameroon in recent years. However, the data on AMR patterns in systematically collected hospital environment from multiple sources is limited. The aim of this study was to describe the pattern of antibiotics resistance and phenotypic characterization of Multidrug resistant bacteria isolates in four hospitals of Littoral region, Cameroon. This approach will be useful to the collection, analysis and sharing of data related to antimicrobial resistance at a global level to inform decision-making, drive local, national and regional action to apply suitable and effective intervention.

\section{MATERIALS AND METHODS}

\section{Study site}

The health facilities were selected using the following criteria: the different technical platform, attendance (social level and frequency), care offered (primary, secondary and central health care according to Cameroon health system) and units. Four hospital facilities (private and public sector) were selected: Saint Jean de Malte Hospital of Njombe (HSJMN), Military Hospital of Douala (MHD), New Bell District Hospital (NBDH) and Medical Health Center of Ndokoti (MHCN) were used for the study.

\section{Study design}

A descriptive, cross-sectional hospital based study was performed. We collected swaps for culture from seven units (Pediatric unit, Medical unit, Operating Theatre, Laboratory, Surgical unit, Emergency and Maternity) specific environmental points (10 different equipment and 10 different materials) in the four hospitals between December
2018 to May 2019, for the selection expected reservoir sample.

\section{Sample collection and technique}

In each health facility, simple random sampling technique was used to collect the sample. Consideration was given to equipment and material with constant hand contacts: 10 equipment : treatment tables, operating tables, delivery tables, office tables, anaesthesia equipment, surgical aspirators, oxygen concentrators, wheelchairs, patients and office chairs. 10 materials: fans, patient bedside tables, patients bed rails, trolleys, door handles, negastoscopes, baby scales, air conditioners, Antiseptic container boxes and Antiseptic container covers. Collection was daily done in the morning after disinfection by hospital workers, but before start of work in each unit. This was done by swabing all selected equipment and materials using sterile cotton tipped applicators moistened in sterile normal saline. These swabs were placed in labelled caps with unit and type of equipment or material identification numbers and immediately transported to the microbiology laboratory for processing.

\section{Laboratory processing}

\section{Inoculation and identification of bacteria species}

Specimens were inoculated directly according to Good Laboratory Practice Guidelines on four agar media consecutively (three sector petri plates with different media: Eosine Methylene blue (EMB), Cystine-lactose-electrolyte deficient agar (CLED), Mannitol salt agar and the blood agar medium in one segmented petri plates) Microxpress ${ }^{\circledR}$ ISO $9001: 2008$ EN ISO $13485: 2012$. After inoculation, the three sectors media were incubated overnight at $37^{\circ} \mathrm{C}$ aerobically for 18 - 24 hours and blood agar medium anaerobically up to a maximum of 48 hours and inspected for bacterial growths. Identification of bacteria to species level was done using colony characteristics, motility test, Gram's staining technique, different biochemical tests comprising of ; oxidase, catalase, coagulase, urease, indole and API Staph, API Strepto and API $20 \mathrm{E}^{\mathrm{TM}}$ BioMerieux according to standard methods ${ }^{26}$.

\section{Antibiotic Susceptibility Testing and MDRB}

The Kirby-Bauer disk-diffusion method (on Mueller Hinton agar) was used for performing antimicrobial susceptibility patterns and reported according to Clinical and Laboratory Standards Institute (CLSI) guidelines ${ }^{27}$. Base on it and also common locally antibiotic used, 18 antibiotics product of (BioMaxima S.A. (POLAND) and Oxoid (UK)) were included: Amoxicilin $(25 \mu \mathrm{g})$, Penicillin G $(10 \mu \mathrm{g})$, Cefoxitin $(30 \mu \mathrm{g})$, Ceftriaxone $(30 \mu \mathrm{g})$, Amoxicillin-clavulanic acid $(20+10 \mu \mathrm{g})$, Ceftazidime $(30 \mu \mathrm{g})$, Aztreonam $(30 \mu \mathrm{g})$, Imipenem $(10 \mu \mathrm{g})$, Vancomycin $(30 \mu \mathrm{g})$, Oxacillin $(1 \mu \mathrm{g})$, Gentamycin $(10 \mu \mathrm{g})$, Amikacin $(30 \mu \mathrm{g}), \quad$ Erythromycin $(15 \mu \mathrm{g}), \quad$ Azithromycin

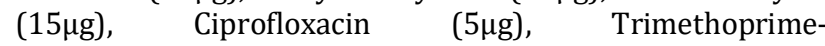
sulfamethoxazole $(25 \mu \mathrm{g})$, Tetracycline $(10 \mu \mathrm{g})$, Clindamycin $(2 \mu \mathrm{g})$. Predominant bacteria and those more likely to cause nosocomial infections (a total of 89 isolated belonging to 13 species) were selected and tested each one with all the 18 antibiotics. Each inoculum was standardized to an optical density of 0.5 McFarland turbidity standard and inoculated by swabbing Mueller Hinton agar plates. The inoculated plates were allowed to stand at room temperature (for no more than 30 minutes) until the moisture in the inoculum spots was absorbed by the agar. The plates were inverted and incubated at $37^{\circ} \mathrm{C}$ for 16 to $20 \mathrm{hr}$. The result of each antibiotic testing was determined in the cases where inhibition zone diameters of strains was within performance ranges according to those recommended by manufacturer's and CLSI guidelines, 201727. 
MDRB were as follows: extended-spectrum beta-lactamase (ESBL)-producin, methicillin-resistant Staphylococcus aureus (MRSA), vancomycin resistant Staphylococcus aureus (VRSA), vancomycin-resistant Enterococcus faecalis (VRE), vancomycin -resistant coagulase negative Staphylococcus (VRCoNS), (MDR) multidrug-resistant and extensive drugresistant (XDR)

\section{Determination of MDRB}

- ESBL was performed using both initial screen test with ideal indicators 3G Cefalosporin discs (Ceftriaxone $30 \mu \mathrm{g}$, Cefoxitin $30 \mu \mathrm{g}$ and Ceftazidime $30 \mu \mathrm{g}$ ) for all the resistant strains as potential ESBL producer and confirmatory test based on double disc synergy test ; The phenotypic confirmation test was performed by using Cefotaxime $30 \mu \mathrm{g}$ or Ceftazidime $30 \mu \mathrm{g}$ both alone and in combination with clavulanic acid $(10 \mu \mathrm{g})$ placed $25 \mathrm{~mm}$ apart (center to center). Inhibition zone of $\geq 5 \mathrm{~mm}$ increase in diameter for antibiotics tested in combination with clavulanic acid versus its zone when tested alone confirms an ESBL producing isolate $27-30$.

- MRSA refered to all $S$. aureus strains resistant both to Oxacillin $1 \mu \mathrm{g}$ and Cefoxitin $30 \mu \mathrm{g}$ indicators discs ${ }^{31,32}$.

- VRSA and VRCoNS defined by S. aureus and coagulase negative Staphylococcus resistant to Vancomycin $30 \mu \mathrm{g}$.

VRE was reported for Vancomycin-resistant Enterococcus ${ }^{33,34}$

- MDR was noted for all the strains resistant to one or more antibiotics to a least two or more antibiotics class as ß-lactams, fluoroquinolone and aminoglycoside except carbapenem 35,36 .

- XDR was indicated by all the strains resistant to two, three or more antibiotics classes including carbapenem 36,37 .

\section{Quality control/ Quality assurance}

Sterility of media was done by incubating one plate from each autoclaved of medium overnight at $35^{\circ}-37^{\circ} \mathrm{C}$ and examine it for contaminants.

Control strains, E. coli ATCC 25922 (non ESBL-producer) and
K. pneumoniae 700603 (ESBL-producer) and Staphylococcus aureus ATCC 25923 (Methicillin sensitive S. aureus) were used to ensure both: Ability to support growth of the target organism(s), ability to produce appropriate biochemical reactions and adequate inhibition zone diameters.

\section{Ethical consideration}

An ethical clearance was obtained from Institutional Ethics committee for research on Human Health of the University of Douala: $\mathrm{N}^{\circ}$ : 1646 IEC-UD/06/2018/T. Also, all afferent administrative authorizations to carry out the project was obtained from the Directors of the four hospitals (HSJMN, NBDH, MHD and MHCN).

\section{Statistical analysis}

Data were recorded in Microsoft Excel 2013 and later transferred to SPSS version 23 software. Categorical variables were summarized using counts, percentages and frequency distribution statistical analysis to reckon the results.

\section{RESULTS}

\subsection{Result of culture and forms of bacteria isolated}

Out of 236 specimens collected from various hospitals, it was observed that majority 119 (50.4\%) showed positive bacteria growth. Among them, 52.1\% (62/119) were Gram positive cocci, 47.9\% (57/119) Gram negative bacilli. 33 different species were found to belong to 5 Gram positif cocci and 28 Gram negative bacilli (13 Enterobacteriaceae and 15 non enterobacteriaceae). In general in Gram positive cocci the most prevalent identified were Staphylococcus aureus (37.8\% (45/119)), while of Gram negative bacilli isolated, majority were non Enterobateriaceae 26.1\% (31/119) with a highest percentage of Acinetobacter spp , Aeromonas hydrophilas and Pasteurella pneumotropica (3.4\% (4/119)), followed by Enterobacteriaceae family 21.9\% (26/119) where E. coli showed a highest percentage $(5.0 \%(6 / 119))$.The above explanation and other detail of bacterial isolates are illustrated in the table 1 below.

Table 1 : Prevalence of isolated bacteria

\begin{tabular}{|c|c|c|c|c|c|c|c|c|c|}
\hline Bacteria & $\mathbf{n}$ & $(\%)$ & Bacteria & $\mathbf{n}$ & (\%) & Bacteria & $\mathbf{n}$ & $(\%)$ & total \\
\hline Gram positive Cocci & 62 & (52.1) & Enterobacteriaceae & 26 & (21.9) & Non Enterobacteriaceae & 31 & (26.1) & $119(100)$ \\
\hline Staphylococcus aureus & 45 & $(37.8)$ & Serratia plymuthica & 2 & $(1.7)$ & Pseudomonas putida & 2 & $(1.7)$ & \\
\hline Micrococcus spp & 3 & $(2.5)$ & Serratia mubidaca & 3 & $(2.5)$ & Pseudomonas fluorescens & 1 & $(0.8)$ & \\
\hline Staphylococcus epidermidis & 10 & $(8.4)$ & serratia liquefasiens & 2 & $(1.7)$ & Pseudomonas mallei & 1 & $(0.8)$ & \\
\hline Staphylococcus saprophyticus & 3 & $(2.5)$ & Serratia cinetica & 1 & $(0.8)$ & Acinetobacter spp & 4 & $(3.4)$ & \\
\hline \multirow[t]{11}{*}{ Enterococcus feacalis } & 1 & $(0.8)$ & Erwinia spp & 2 & $(1.7)$ & Acinetobacter bomannii & 3 & $(2.5)$ & \\
\hline & & & Escherichia coli & 6 & $(5.0)$ & Areomonas hydrophilas & 4 & $(3.4)$ & \\
\hline & & & Enterobacter agglomerans & 3 & $(2.5)$ & Pasteurella pneumotropica & 4 & $(3.4)$ & \\
\hline & & & Enterobacter sakazaki & 1 & $(0.8)$ & Chryseomonas meningoseptica & 2 & $(1.7)$ & \\
\hline & & & Proteus mirabilis & 1 & $(0.8)$ & Mannheimia haemolitica & 2 & $(1.7)$ & \\
\hline & & & Priovidencia stuartii & 1 & $(0.8)$ & Xantomonas maltophila & 1 & $(0.8)$ & \\
\hline & & & Salmonella arizona & 1 & $(0.8)$ & Plesiomonas shigelloides & 1 & $(0.8)$ & \\
\hline & & & Salmonella spp & 2 & $(1.7)$ & Chyseomonas putida & 1 & $(0.8)$ & \\
\hline & & & Serratia odorifera & 1 & $(0.8)$ & Alcaligenes spp & 2 & $(1.7)$ & \\
\hline & & & & & & Pantoa spp3 & 2 & $(1.7)$ & \\
\hline & & & & & & Burkholderia ceptica & 1 & $(0.8)$ & \\
\hline
\end{tabular}


Antibiotic resistance profile of the most prevalent bacterial isolates and those related to NIs

Among the119 bacteria (33 different species) isolates, 89 (13 species), based on predominant bacteria and those more likely to cause nosocomial infections were selected and tested each to 18 antibiotics classified in four groups depending on their most target site and mechanism of action in bacteria structure ("group 1": cell wall and/or cell membrane synthesis inhibitors, "group 2": Protein synthesis inhibitors, "group 3": Nucleic acid inhibitors and "group 4": Folic acid synthesis inhibitor) ${ }^{38 .}$

Regarding the global profile of resistance, there were high level of resistance to most of the antibiotics of "group 1" especially Penicillin (amoxicillin (77.5 \%) and Oxacillin (76.4\%)), followed by 3GCephalosporine (Ceftazidime $(74.2$ $\%)$ and Monobactam (Aztreonam (70.8\%)). Although the least level of resistance was observed in the same "group 1" concerning Carbapenem (imipenem (5.6\%)).
Looking at the most common prevalent bacteria tested, in Gram positif cocci, $S$. aureus was the only species that showed resistance to all antibiotic tested independently of the group, most predominant in group 1 to oxacillin $91.1 \%$.

In general among Gram negative bacilli, all the most common isolated were all resistant $(100 \%)$ to one or more tested antibiotics: Acinetobacter spp and Aeromonas hydrophilas both into "group 1" but two different class of antibiotics, especially to Penicillin (Amoxicillin) and Glycopeptide (Vacomycin) . Pasteurella pneumotropica into"group 1and 2"respectively to 3GCephalosporin (Ceftriaxone) and Macrolide (Azytromycin). E. coli only into "group 1" to 2GCephalosporin (Cefoxitin). Nevertheless, all were susceptible (100\%) only into "group 1" as well as one class, particulally to Carbapenem (Imipenem), except Pasteurella pneumotropica with one strain resistant to Carbapenem (Imipenem). Details of these are depicted in Tables 2, 3 and 4 bellow.

Table 2: Antibiotic resistance patterns of major isolated bacteria species to different antibiotics of “Group 1"

(Cell wall and or cell membrane synthesis inhibitors)

\begin{tabular}{|c|c|c|c|c|c|c|c|c|c|c|c|}
\hline \multirow[b]{3}{*}{ Name of bacteria } & \multirow[b]{3}{*}{ Total } & \multicolumn{10}{|l|}{ Group 1} \\
\hline & & \multicolumn{10}{|c|}{ B-lactamin (cell wall and/ or cell membrane growth inhibitors) } \\
\hline & & $\begin{array}{l}\text { AMX } \\
\text { n(\%) }\end{array}$ & $\begin{array}{l}\text { PEN } \\
\text { n(\%) }\end{array}$ & $\begin{array}{l}\text { FOX } \\
\text { n(\%) }\end{array}$ & $\begin{array}{l}\text { CRO } \\
\text { n(\%) }\end{array}$ & $\begin{array}{l}\text { AMC } \\
\text { n(\%) }\end{array}$ & $\begin{array}{l}\text { CAZ } \\
\text { n(\%) }\end{array}$ & $\begin{array}{l}\text { ATM } \\
\text { n (\%) }\end{array}$ & $\begin{array}{l}\text { IMP } \\
\text { n (\%) }\end{array}$ & $\begin{array}{l}\text { VA } \\
\text { n(\%) }\end{array}$ & $\begin{array}{l}\text { OX } \\
\text { n(\%) }\end{array}$ \\
\hline Staphylococcus aureus & 45 & $33(73.3)$ & $36(80)$ & $37(82.2)$ & $32(71.1)$ & 20(44.4) & $40(88.9)$ & $42(93.3)$ & $4(8.9)$ & $18(40)$ & $41(91.1)$ \\
\hline Staphylococcus epidermidis & 10 & $9(90)$ & $4(40)$ & $5(50)$ & $6(60)$ & $4(40)$ & $7(70)$ & $7(70)$ & 0 & $4(40)$ & $7(70)$ \\
\hline Aeromonas hydrophilas & 4 & $4(100)$ & $1(25)$ & $1(25)$ & $2(50)$ & 0 & $2(50)$ & $1(25)$ & 0 & $4(100)$ & $1(25)$ \\
\hline Serratia mubidaca & 3 & $2(66.7)$ & $3(100)$ & $2(66.7)$ & $2(66.7)$ & $2(66.7)$ & 0 & $2(66.7)$ & 0 & $1(33.4)$ & 0 \\
\hline Erwinia spp & 2 & $2(100)$ & 0 & 0 & $2(100)$ & $2(100)$ & $2(100)$ & 0 & 0 & $2(100)$ & $1(50)$ \\
\hline Acinetobacter spp & 4 & $4(100)$ & $4(100)$ & $1(25)$ & $1(25)$ & $2(50)$ & $2(50)$ & $1(25)$ & 0 & $4(100)$ & $4(100)$ \\
\hline Escherichia coli & 6 & $4(66.7)$ & $3(50)$ & 4 & $1(16.7)$ & $2(33.3)$ & $2(33.3)$ & $1(16.7)$ & 0 & $3(50)$ & $2(33.3)$ \\
\hline Pasteurella pneumotropica & 4 & $3(75)$ & $1(25)$ & $3(75)$ & $4(100)$ & $3(75)$ & $3(75)$ & $3(75)$ & $1(25)$ & $3(75)$ & $3(75)$ \\
\hline Enterobacter agglomerans & 3 & $2(66.7)$ & $2(66.7)$ & 0 & $2(66.7)$ & 0 & $2(66.7)$ & $2(66.7)$ & 0 & $2(66.7)$ & $2(66.7)$ \\
\hline Salmonella spp & 2 & 0 & $1(50)$ & $1(50)$ & $1(50)$ & 0 & 0 & $1(50)$ & 0 & 0 & 0 \\
\hline Acinetobacter bomanii & 3 & $3(100)$ & $2(66.7)$ & $2(66.7)$ & $3(100)$ & $2(66.7)$ & $3(100)$ & $2(66.7)$ & 0 & $2(66.7)$ & $3(100)$ \\
\hline Enterococcus feacalis & 1 & $1(100)$ & $1(100)$ & $1(100)$ & $1(100)$ & 0 & $1(100)$ & $1(100)$ & 0 & $1(100)$ & $1(100)$ \\
\hline Pseudomonas putida & 2 & $2(100)$ & $2(100)$ & $2(100)$ & $2(100)$ & 0 & $2(100)$ & 0 & 0 & $2(100)$ & $2(100)$ \\
\hline Total & 89 & $69(77.5)$ & $60(67.4)$ & $59(66.3)$ & $58(65.16)$ & $37(41.6)$ & $66(74.2)$ & $63(70.8)$ & $5(5.6)$ & $46(51.7)$ & $67(75.3)$ \\
\hline
\end{tabular}

AMX: Amoxicilin; PEN : Penicillin G; FOX:Cefoxitin; CRO : Ceftriaxone; AMC : Amoxicillin-clavulanic acid ; CAZ : Ceftazidime ; ATM : Aztreonam; IMP : Imipenem; VA : Vancomycin ; OX: Oxacillin; 
Table 3: Antibiotic resistance patterns of major isolated bacteria species to different antibiotics of “Group 2" (Protein synthesis inhibitors)

\begin{tabular}{|c|c|c|c|c|c|c|}
\hline \multirow[b]{3}{*}{ Name of bacteria } & \multirow[b]{3}{*}{ Total } & \multicolumn{5}{|c|}{ Group 2} \\
\hline & & \multicolumn{5}{|c|}{ Protein synthesis inhibitors } \\
\hline & & $\begin{array}{l}\text { CN } \\
\text { n (\%) }\end{array}$ & $\begin{array}{l}\text { AK } \\
\text { n (\%) }\end{array}$ & $\begin{array}{l}\text { E } \\
\text { n (\%) }\end{array}$ & $\begin{array}{l}\text { AZT } \\
\text { N (\%) }\end{array}$ & $\begin{array}{l}\text { CL } \\
\text { n (\%) }\end{array}$ \\
\hline Staphylococcus aureus & 45 & $14(31.1)$ & 13 (28.9) & $22(48.8)$ & $37(82.2)$ & $10(22.2)$ \\
\hline Staphylococcus epidermidis & 10 & $2(20)$ & $5(50)$ & $6(60)$ & $6(60)$ & $4(40)$ \\
\hline Aeromonas hydrophilas & 4 & 0 & 0 & $1(25)$ & $1(25)$ & $1(25)$ \\
\hline Serratia mubidaca & 3 & $1(33.3)$ & $1(33.3)$ & $1(33.3)$ & $1(16.7)$ & $1(33.7)$ \\
\hline Erwinia spp & 2 & 0 & 0 & $1(50)$ & 0 & $1(50)$ \\
\hline Acinetobacter spp & 4 & 0 & 0 & $1(25)$ & $1(25)$ & $1(25)$ \\
\hline Escherichia coli & 6 & 0 & $2(33.3)$ & $3(50)$ & $1(16.7)$ & $1(16.7)$ \\
\hline Pasteurella pneumotropica & 4 & $2(50)$ & $2(50)$ & $3(75)$ & $4(100)$ & $2(50)$ \\
\hline Enterobacter agglomerans & 3 & $2(66.7)$ & 0 & 0 & $3(100)$ & 0 \\
\hline Salmonella spp & 2 & 0 & 0 & 0 & $1(50)$ & $1(50)$ \\
\hline Acinetobacter bomanii & 3 & 0 & 0 & $2(66.7)$ & $1(33.3)$ & $2(66.7)$ \\
\hline Enterococcus feacalis & 1 & 0 & 0 & 0 & $1(100)$ & $1(100)$ \\
\hline Pseudomonas putida & 2 & $1(50)$ & $1(50)$ & $2(100)$ & $2(100)$ & $2(100)$ \\
\hline Total & 89 & $22(24.7)$ & $24(26.9)$ & $42(47.2)$ & $60(67.4)$ & $27(30.3)$ \\
\hline
\end{tabular}

CN: Gentamycin; AK: Amikacin; E: Erythromycin; AZT: Azithromycin; CL: Clindamycin

Table 4: Antibiotic resistance patterns of major isolated bacteria species to different antibiotics of "Group 3 and 4" (nucleic acid synthesis inhibitors and folic acid synthesis inhibitor)

\begin{tabular}{|c|c|c|c|c|}
\hline \multirow[b]{3}{*}{ Name of bacteria } & \multirow[b]{3}{*}{ Total } & \multicolumn{2}{|c|}{ Groupe 3} & \multirow{3}{*}{\begin{tabular}{l}
\multicolumn{1}{c}{ Groupe 4 } \\
Folic acid synthesis inhibitors \\
SXT \\
n (\%) \\
\end{tabular}} \\
\hline & & \multicolumn{2}{|c|}{ Nucleic acid synthesis inhibitors } & \\
\hline & & $\begin{array}{l}\text { CIP } \\
\text { n (\%) }\end{array}$ & $\begin{array}{l}\text { TE } \\
\text { n (\%) }\end{array}$ & \\
\hline Staphylococcus aureus & 45 & $14(31.1)$ & $14(31.1)$ & $22(48.9)$ \\
\hline Staphylococcus epidermidis & 10 & $2(20.0)$ & $1(10)$ & $5(50)$ \\
\hline Aeromonas hydrophilas & 4 & $2(50)$ & $2(50)$ & $4(100)$ \\
\hline Serratia mubidaca & 3 & $1(33.3)$ & $1(33.3)$ & $1(33.3)$ \\
\hline Erwinia spp & 2 & 0 & 0 & $1(50)$ \\
\hline Acinetobacter spp & 4 & $1(25)$ & $2(50)$ & $2(50)$ \\
\hline Escherichia coli & 6 & $1(16.7)$ & $3(50)$ & $3(50)$ \\
\hline Pasteurella pneumotropica & 4 & $2(50)$ & $3(75)$ & $3(75)$ \\
\hline Enterobacter agglomerans & 3 & $2(66.7)$ & 0 & $2(66.7)$ \\
\hline Salmonella spp & 2 & 0 & 0 & $1(50)$ \\
\hline Acinetobacter bomanii & 3 & $1(33.3)$ & $2(66.7)$ & $2(66.7)$ \\
\hline Enterococcus feacalis & 1 & 0 & $1(100)$ & $1(100)$ \\
\hline Pseudomonas putida & 2 & $2(100)$ & $2(100)$ & $2(50)$ \\
\hline Total & 89 & $28(31.5)$ & $31(34.8)$ & $49(55.1)$ \\
\hline
\end{tabular}

CIP: Ciprofloxacin; TE: Tetracycline; SXT: Trimethoprime-sulfamethoxazole;

\section{Common MDRB detected}

Common type of MDRB according to most common isolated bacteria and those at risk to causing NIs

A total of 119 bacteria (33 different species) were isolated in wich 89 (13 species) were selected and tested each one to 18 antibiotics. Of all 89 strains, 11 different species showed more than the half 56 considered MDRB, giving the overall prevalence of $62.9 \%$.
Serratia mubidaca (3 strains) and salmonelle spp (2 strains) did not show any type of MDRB.

Regarding the general profile of 89 tested strains, MRSA were the mostly detected $57.5 \%$ (30/89), followed by ESBL $10.1 \%(9 / 89)$. The lowest percentage was recorded by VRE and XDR both with $1.1 \%(1 / 89)$. According to the type of MDRB in selected isolated bacteria, all the strains (100\%) of 2 species, especially A. hydrophilas, and E. feacalis were characterized MDRB. However, S. aureus strains reported 
significant rate of MDRB $84.4 \%(38 / 45)$ and recorded $4.4 \%$ (2/45) strains of ESBL. P. pneumotropica provided $75 \%$ (3/4), as well as the only Gram negative bacilli specie where one strain was resistant to more than three different class of tested antibiotics included Carbapenem (imipenem) and consequently named XDR with $25 \%(1 / 4)$. Table 5 below show a detailed illustration of the above explanation.

Table 5: Common type MDRB profiles of tested bacteria isolates

\begin{tabular}{|c|c|c|c|c|c|c|c|c|c|}
\hline \multirow[b]{2}{*}{ Name of bacteria } & \multicolumn{7}{|c|}{ Common type MDRB } & \multicolumn{2}{|c|}{ Total } \\
\hline & $\begin{array}{l}\text { ESBL } \\
\text { n (\%) }\end{array}$ & $\begin{array}{l}\text { MRSA } \\
\text { n (\%) }\end{array}$ & $\begin{array}{l}\text { VRE } \\
\text { n (\%) }\end{array}$ & $\begin{array}{l}\text { MDR } \\
\text { n (\%) }\end{array}$ & $\begin{array}{l}\text { XDR } \\
\text { n (\%) }\end{array}$ & $\begin{array}{l}\text { VRSA } \\
\text { n (\%) }\end{array}$ & $\begin{array}{l}\text { VRCoNS } \\
\text { n (\%) }\end{array}$ & $\begin{array}{l}\text { of MDRB } \\
\text { n (\%) }\end{array}$ & $\begin{array}{l}\text { Isolated } \\
\text { bacteria }\end{array}$ \\
\hline Staphylococcus aureus & $2(4.4)$ & $30(66.7)$ & 0 & 0 & 0 & $6(13.3)$ & 0 & $38(84.4)$ & 45 \\
\hline Staphylococcus epidermidis & 0 & 0 & 0 & 0 & 0 & 0 & $3(30.0)$ & $3(30.0)$ & 10 \\
\hline Aeromonas hydrophilas & $3(75.0)$ & 0 & 0 & $1(25.0)$ & 0 & 0 & 0 & $4(100.0)$ & 4 \\
\hline Erwinia spp & $1(50.0)$ & 0 & 0 & 0 & 0 & 0 & 0 & $1(50)$ & 2 \\
\hline Acinetobacter spp & 0 & 0 & 0 & $1(25.0)$ & 0 & 0 & 0 & $1(25.0)$ & 4 \\
\hline Escherichia coli & $1(16.7)$ & 0 & 0 & 0 & 0 & 0 & 0 & $1(16.66)$ & 6 \\
\hline Pasteurella pneumotropica & $1(25.0)$ & 0 & 0 & $1(25.0)$ & $1(25.0)$ & 0 & 0 & $3(75.0)$ & 4 \\
\hline Enterobacter agglomerans & 0 & 0 & 0 & $1(33.3)$ & 0 & 0 & 0 & $1(33.33)$ & 3 \\
\hline Acinetobacter bomanii & $1(33.3)$ & 0 & 0 & $1(33.3)$ & 0 & 0 & 0 & $2(66.66)$ & 3 \\
\hline Enterococcus feacalis & 0 & 0 & $1(100.0)$ & 0 & 0 & 0 & 0 & $1(100.0)$ & 1 \\
\hline Pseudomonas putida & 0 & 0 & 0 & $1(50.0)$ & 0 & 0 & 0 & $1(50.0)$ & 2 \\
\hline NA & 0 & 0 & 0 & 0 & 0 & 0 & 0 & 0 & 5 \\
\hline Total & $9(10.1)$ & $30(33.7)$ & $1(1.1)$ & $6(6.7)$ & $1(1.1)$ & $6(6.7)$ & $3(3.37)$ & $56(62.92)$ & 89 \\
\hline
\end{tabular}

NA: none applicable (Serratia mubidaca and salmonelle spp without MDRB)

* Frequency calculated based on type of MDRB detected in each species

ESBL: extended-spectrum beta-lactamase; MRSA: methicillin resistant Staphylococcus aureus

VRSA: vancomycin resistant Staphylococcus aureus; VRCoNS: vancomycin-resistant coagulase negative Staphylococcus

VRE: vancomycin-resistant Enterococcus

MDR: multidrug-resistant (to one or more antibiotics to a least two or more antibiotics class as ß-lactamin, fluoroquinolone and aminoglycoside except carbapenem)

XDR: extensively drug-resistant (resistant to two or more antibiotics classes including carbapenem)

\section{Common type MDRB according to hospital facilities}

Out of 56 MDRB detected, MHD shelter the highest percentage $39.3 \%(22 / 56)$ as well as the only hospital where a single strains of XDR was isolated 1.8\% (1/56). Followed by NBDH $28 \%(16 / 56)$, both mainly constituting of MRSA, respectively $25 \%(14 / 56)$ and $12.5 \%(7 / 56)$. In addition, it's necessary to notice that, ESBL, the most second MDRB type isolated, were observed in three hospitals (NBDH, MHCN and MHD) with equal level 5.4\% (3/56). However, MRSA, the predominant MDRB type was detected in all the four hospitals facilities. Details are tabulated in Table 6 below.

Table 6: Distribution of common MDRB per hospital facilities

\begin{tabular}{lllllc}
\hline & \multicolumn{5}{c}{ Hospital facilities } \\
\cline { 2 - 6 } Common MDRB & NBDH & HSJMN & MHCN & MHD & Total \\
\hline ESBL & $\mathbf{n}(\%)$ & $\mathbf{n}(\%)$ & $\mathbf{n}(\%)$ & $\mathbf{n}(\%)$ & $\mathbf{n}(\%)$ \\
MRSA & $3(5.4)$ & 0 & $3(5.4)$ & $3(5.4)$ & $9(16.1)$ \\
VRE & $7(12.5)$ & $7(12.5)$ & $2(3.6)$ & $14(25)$ & $30(53.6)$ \\
MDR & $1(1.8)$ & 0 & 0 & 0 & $1(1.8)$ \\
XDR & 0 & $3(5.4)$ & $1(1.8)$ & $2(3.6)$ & $6(10.7)$ \\
VRSA & 0 & 0 & 0 & $1(1.8)$ & $1(1.8)$ \\
VRCoNS & $4(7.1)$ & 0 & $1(1.8)$ & $1(1.8)$ & $6(10.7)$ \\
Total & $1(1.8)$ & $1(1.8)$ & 0 & $1(1.8)$ & $3(5.4)$
\end{tabular}

ESBL: extended-spectrum beta-lactamase; $\quad$ MRSA: methicillin resistant Staphylococcus aureus

VRSA: vancomycin resistant Staphylococcus aureus; VRCoNS: vancomycin -resistant coagulase negative Staphylococcus

VRE: vancomycin -resistant Enterococcus

MDR: multidrug -resistant (to one or more antibiotics to a least two or more antibiotics class as ß-lactamin, fluoroquinolone and aminoglycoside except carbapenem)

XDR: extensively drug-resistant (resistant to two or more antibiotics classes including carbapenem) 


\section{Common type MDRB according to hospital units}

Of all 56 MDRB detected, Emergency unit was more contaminated $17.9 \%(10 / 56)$ in which MRSA was overlooked $10.7 \%(6 / 56)$. This was follow by Pediatric unit, Medical unit and maternity all with equal level of $16.1 \%$
(9/56). While laboratory reported the lowest with $8.9 \%$ (5/56). Likewise, in hospital facilities, MRSA was overlooked and present in all units. Nevertheless, the only one strain $1.8 \%(1 / 56)$ VRE was found in Medical unit the same as single strain $1.8 \%(1 / 56)$ of XDR recorded in Pediatric unit.

Table 7: Distribution of MDRB type per hospital units

\begin{tabular}{|c|c|c|c|c|c|c|c|c|}
\hline \multirow[b]{3}{*}{ Common } & \multicolumn{2}{|c|}{ Pediatric } & \multirow{3}{*}{$\begin{array}{l}\text { Operating } \\
\text { Theatre }\end{array}$} & \multirow{3}{*}{ Laboratory } & \multicolumn{2}{|r|}{ Emergency } & \multirow{3}{*}{ Maternity } & \multirow{3}{*}{ TOTAL } \\
\hline & unit & Medical unit & & & Surgical unit & unit & & \\
\hline & & & & & & & & \\
\hline MDRB & n (\%) & n (\%) & n (\%) & n (\%) & n (\%) & n (\%) & n (\%) & n (\%) \\
\hline ESBL & $3(5.4)$ & $2(3.6)$ & 0 & $1(1.8)$ & $1(1.8)$ & $2(3.6)$ & 0 & $9(16.1)$ \\
\hline MRSA & $4(7.1)$ & $4(7.1)$ & $5(8.9)$ & $3(5.4)$ & $3(5.4)$ & $6(10.7)$ & $5(8.9)$ & $30(53.6)$ \\
\hline VRE & 0 & $1(1.8)$ & 0 & 0 & 0 & 0 & 0 & $1(1.8)$ \\
\hline MDR & $1(1.8)$ & 0 & $2(3.6)$ & $1(1.8)$ & $1(1.8)$ & $1(1.8)$ & 0 & $6(10.7)$ \\
\hline XDR & $1(1.8)$ & 0 & 0 & 0 & 0 & 0 & 0 & $1(1.8)$ \\
\hline VRSA & 0 & $2(3.6)$ & $1(1.8)$ & 0 & $1(1.8)$ & $1(1.8)$ & $1(1.8)$ & $6(10.7)$ \\
\hline VRCoNS & 0 & 0 & 0 & 0 & 0 & 0 & $3(5.4)$ & $3(5.4)$ \\
\hline Total & $9(16.1)$ & $9(16.1)$ & $8(14.3)$ & $5(8.9)$ & $6(10.7)$ & 10 (17.9) & $9(16.1)$ & $56(100.0)$ \\
\hline
\end{tabular}

ESBL : extended-spectrum beta-lactamase ;

MRSA: methicillin resistant Staphylococcus aureus

VRSA : vancomycin resistant Staphylococcus aureus ; VRCoNS: vancomycin -resistant coagulase negative Staphylococcus

VRE: vancomycin -resistant Enterococcus

MDR: multidrug-resistant (to one or more antibiotics to a least two or more antibiotics class as ß-lactamin, fluoroquinolone and aminoglycoside except carbapenem)

XDR: extensively drug -resistant (resistant to two or more antibiotics classes including carbapenem)

\section{DISCUSSION}

One of the major role of the clinical microbiology laboratory is to provide susceptibility testing data that can serve both as a guide to clinicians for effective therapy and however in the presence of multidrug-resistant organisms (MDROs), can be helpful to initiate early and appropriate investigation to prevent outbreak-related isolates. Moreover, effective targeted prevention has revolutionized the efforts to control the global crisis of NIs and identifies key factors influencing the emergence been of AMR patterns ${ }^{39-}{ }^{41}$. In addition, as Center of Disease Control (CDC) reported in September 2019, to slow AMR, the Challenge resulted in more than 350 organizations across the globe ${ }^{42}$. Therefore, one critical area may require immediate attention for rational decision in the management of antimicrobial use and infection control policy.

\section{Prevalence of isolated bacteria}

In this study, the overall prevalence of surface bacteria contamination was $50.4 \%(119 / 236)$. This is higher than $45.6 \%$ and $43.8 \%$ culture-positive bacteria from 57 surface sampled by Costa et al.(2019) in intensive care unit and Worku et al.(2018) from hospital devices of Mizan-Tepi University Teaching Hospital in Ethiopia, respectively43, 44. Nevertheless, $50.4 \%$ is significantly lower than what was recovered in several studies: Gonsu $\mathrm{KH}$ et al. YaoundéCameroon 2015(98.6\%), Fotsing et al. Baganté-Cameroon 2020 (95\%), Bhatta et al. Pokhara-Nepal 2018 (78\%), Aseer et al. Arba Minch-Ethiopia 2019 (71, 7 \%), Ndu et al. EnuguNigeria 2019 (70.3\%) and Afleet al. Abomey-Benin 2019 $(65 \%)^{2}, 45-49$. The justification of the difference might be related to various factors including: level of the target setting, target sample, sample size, decontamination systems gaps, but most likely to the type of bacteria isolated (Only Gram positive cocci and Gram negative rods in our study versus all type of bacteria including Gram positive rods in those several studies. Looking at the type of bacteria found, the $52.1 \%(64 / 119)$ Gram positive cocci and $47.9 \%$ (57/119) Gram negative bacilli (Table 1), are consistent with those reported in recent studies ${ }^{2}, 8,44,45$. This higher prevalence of Gram-positive bacteria may be explained, by the chemical composition of this bacterial cell envelops that provide resistance to harsher environmental conditions than Gram-negatives ${ }^{45}$. On the contrary, amongst culture positive infections, Gram-negative organisms predominated (13/16 (81.3\%)), similar to Hearn et al. (2017), who prospectively determinate HAI incidence in a Cambodian pediatric referral hospital50. Our study also revealed that, Staphylococcus aureus was the predominant isolate bacteria $37.8 \%$ (45/119). This was similar to the findings of a several studies $45,46,48,51$. Thus, $S$. aureus have relative resistance to common disinfectant and consequently predilection to persist in drying surface. This can be supported by the study of Suleyman et al. (2018). Regarding the survival times of nosocomial pathogens on environmental surfaces, $S$. aureus can resist and live up to 5 years in a dry inanimate surface ${ }^{52}$. Otherwise, our results are contrary to the findings of Agaba et al. (2017) and Nouri et al. (2020) whose predominant isolates was found to be Klebsiella $s p p^{53,54}$ and also at variance to the work of Gonsu et al. ${ }^{2}$ in two referral hospitals Yaoundé-Cameroon. In General, as prominent isolated bacteria $S$. aureus was followed by E. coli $(5.04 \%$ (6/119)) and Acinetobacter spp (3.36\% (4/119)). A recent study (2018) had shown the predominance of $S$. aureus, E. coli and Acinetobacter species, in the bacteriological profile of frequently touched objects in a tertiary care hospital of Pokhara, Nepal46, as well as these bacteria are well-knowed and recognized like common nosocomial organisms ${ }^{53-55}$. However, Staphylococcus and E. coli are facultative aerobes, then ubiquitous, as well as Acinetobacter spp, Gram negative and non-fermenting coccobacilli are ubiquitous $45,56$. 
Furthermore, it had been demonstrated that the organisms from surface of frequent contact in hospital are likely to get transmitted to healthcare workers, patients and visitors ${ }^{9,43}$, 46. In addition, as such, previously in our recent article we suggested that two of our major isolates $(S$. aureus and Acinetobacter spp), belongs to the three of the priority pathogens on the WHO list for Global Antimicrobial Resistance Surveillance System (GLASS) reporting, namely Klebsiella pneumonia, Acinetobacter baumannii and Staphylococcus aureus to cause HAIs ${ }^{57}$. Thus, recent studies have demonstrated that our prominent isolated bacteria $(S$. aureus, Acinetobacter spp and E. coli) propensity to cause outbreaks is facilitated by antimicrobial resistance and their ability to resist desiccation and survive for prolonged periods on multiple environmental surfaces $52,58-61$.

Antibiotic resistance profile of the most prevalent bacterial isolates and those related to NIs

Regarding the global profile of resistance, over the 13 species (89 strains) tested through 18 antibiotics, there was a high level of resistance to most of the antibiotics of "group 1" especially Penicillin (amoxicillin (77.5\%) and Oxacillin (76.4\%)), followed by 3GCephalosporine (Ceftazidime (74.2\%) and Monobactam (Aztreonam (70.8\%)). Although, observed in the same "group 1", Carbapenem (imipenem (5.61\%)) was the lowest except among Pasteurella pneumotropica with one strain resistant. Similar results are reported (2020) by the Canadian Nosocomial Infection Surveillance Program where the highest non-susceptibility antibiotics rates were in Penicillin class (ampicillin (43.0\%)) and low than $92.8 \%$ resistant to Cephalosporine (Cefuroxime) reported by Makanjuola et al (2018), while showed all low resistance for Carbapenem (meropenem $(0.4 \%)$,Ertapenem (0.2\%)) and despite low than meropenem $21.4 \%$ respectively) ${ }^{62,17}$. The main isolate of Enterobacteriaceae family, E.coli was $100 \%$ sensitive to Carbapenem (imipenem) as per Chelliah et al with 100\% E. coli 63 .

\section{Common type MDRB profile of different tested isolated bacteria}

Looking at the type of MDRB, the overall prevalence was $62.9 \%$ (56/89) in which MRSA were predominantly $33.7 \%$ (30/89) detected in all hospital facilities and all units). Our results are higher than $58 \%$ obtained by Agada et al. 53 Looking for Nosocomial bacterial infections and their antimicrobial susceptibility patterns among patients in a Ugandan intensive care unit. This difference can be explained by various factors include the target site (single center versus multi center) and sample collection, (clinical \& surface sample). However, it is known that environmental bacterial are more likely to develop resistance than those of clinical sample64. This can be supported by the study of Tan et al. where the target MDROs were recovered from $79 \%$ of sampled surfaces, predominantly MRSA (74 \% of all tested surfaces) ${ }^{65}$. Over $45 \mathrm{~S}$. aureus isolates in this study, $66.7 \%$ (30/45) were MRSA. Our findings are high than those reported by Bhatta et al in a tertiary care hospital of Pokhara, Nepal, where, on $44 \mathrm{~S}$. aureus isolates as predominant bacteria, less than the half $36.3 \%(16 / 44)$ were MRSA 46 . Additionally, likewise it is high compared to $20 / 47$ (44.7\%) MRSA detected by Chaoui et al 64 and $31.25 \%$ according to Chailliah et al. 63. Otherwise in our study ESBL was the second predominant MRDB detected 9/56 (16.07\%). Despite this, we did not confirm this producing enzyme within a MIC or genetic method, our results can be support by those reported by Teghonon et al. (February 2020), in a study looking for these enzymes in the hospitals of the Littoral region, Cameroon ${ }^{66}$. ESBL in S. aureus $4.4 \%$
(2/45) strains are consistent with those of Olutola et al. in 2016, focused on "ESBL detection Antibiotic Susceptibility Profile of Staphylococcus aureus Strains Isolated from Surgical Wounds where ESBL phenotypically expressed in two isolates 24 . This can be support by the fact that, Extended Spectrum Beta-Lactamase (ESBL) capable of hydrolyze penicillins and are often located on plasmids that are transferable from strain to strain and between bacterial species $^{28,} 67$. The role of selective pressures in the environment as well as the medical use of antimicrobials together with the interplay of various genetic mechanisms for horizontal gene transfer are considered and well demonstrated ${ }^{68}$. Additionally, a particular ESBL enzyme, called CTX-M, appears to be spreading in the United States and around the world. The CTX-M enzyme can be shared through DNA (genes) between different Enterobacteriaceae species ${ }^{30}$. XDR is a worrying crisis in the fact that Carbapenems are one of the few remaining antibiotics that can treat ESBL-producing germs ${ }^{30}$. As concerned Salmonella arizona isolates in this study, didn't showed any MDR. On contrary, a review and a meta-analysis study carry out in Cameroon on one health perspective reported Salmonella $s p p$ in animals with a MDR rate of $46.2 \%{ }^{41}$. This difference is evident due to the type of specimen (hospital environment \& animal). Therefore, this high rate of MDR salmonella should be takled with more consideration; consequently it can be shared within short or long-term to human. This is support by the fact that drug resistance in gram-negative bacterial hospital-acquired infections (GNB HAIs) has become ubiquitous in recent years ${ }^{28}$.The dry surface biofilms persist for long period and have been shown as a horizontal transmission driver of (MDROs) 43.

\section{Common types MDRB according to hospital facilities and units}

MHD shelter the highest percentage 39.3\% (22/56), followed by NBDH $28 \%(16 / 56)$, both mainly constituting of MRSA, respectively $25 \%(14 / 56)$ and $12.5 \%(7 / 56)$ as well as the only type present in all hospital facilities and units. Thus, in our previous publication as suggested to explain the distribution of $S$. aureus to all hospitals facilities and units, we expected to observe a higher percentage of MRSA in all hospitals and services due to an outbreak and spread, supported by a study that reported the presence and increase of MRSA in Many hospitals of several countries in Africa $^{57}$. In addition, $S$. aureus have the ability to form biofilm (surface - attached communities of cells) facilitating their persistence in hospital devices and allowing their survival in high antimicrobial concentrations ${ }^{69}$. In fact, $S$. aureus are caractirized by the best biofilm production adhesine that include: Microbial Surface Components Recognizing Adhesive Matrix Molecules (MSCRAMMs) and Polysaccharide Intercellular Adhesine (PIA) due to the presence of several genes: (Fibronectin-binding protein $\mathrm{A}$ (fnbA), intracellular adhesine (icaA, B, C, D) and accessory gene regulator (agrI,II,III,IV)) 70. Consequently, as a healthcare professional, this is the highest risk of horizontal transmission to NIs in risky population, hospitalize patients, healthcare workers, administration staff, and visitors that are expose within themselves 9.

Comparing the units, the Emergency unit was the MDRBs dominantly contaminated area $17.9 \%(10 / 56)$. It is evident and largely known that many factors influencing the development of antimicrobial resistance in this unit include intensive care, immunosuppressive drugs, over consumption of antimicrobial and irrational use of antimicrobial therapy that contribute to selection pressure 14,71 . Otherwise, Medical and Pediatric unit were the second contaminated with $16.1 \%(9 / 56)$, as well as the units where single strain of VRE 
and XDR both $1.8 \%(1 / 56)$ were recorded. Prolongation stay of patient in these current hospitalization units, influencing selection pressure of antimicrobial resistance and can be explained by the emergence of new resistance strains that contributes to XDR within VRE. This can be supported by the study of Soltani et al. (2016) showing that the pediatric unit is the most contaminated area $52 \%{ }^{72}$. However, our VRE percentage were low (1.8\%) compared to $18.7 \%$ and $63 \%$ of Juarez et al. (2015) and Agegne et al. (2018) respectively. Enterococci, especially feacalis species, are well known antibiotic-resistant opportunistic pathogens commonly recovered from patients who received multiple courses of antibiotics and hospitalized for prolonged periods ${ }^{36,73}$.

Finally, the results of our study could serve as a timely regional data of hospital surface epidemiological surveillance basis on which preventive strategy of HAIs and AMR should be built accompanied by active methods of supervisions aimed at improving the safety of health personnel, patients, and visitors. Worrying, such higher and various MDRB hospital surface contaminants area consequence of inadequate and ineffective containment and control measures (cleaning, disinfection and sterilization), in Low-and Middle -Income countries. Many factors account for this: waste disposal, poor infrastructure, insufficient equipment, understaffing, overcrowding, intensive and irrational use of antibiotics, lack or poor antimicrobial stewardship programs, paucity of procedure, briefs poor knowledge, attitude and practices of basic infection prevention control measures 50,74 . Each hospital should be able to develop consistent and locally rapid detection means, recognize trends, report and publish susceptibility patterns to hospital leaders that can help to increase adherence to policies, especially regarding it specific barrier precautions. This will go a long way to reduce the AMR (including HAIs) rate, causing substantial increase in healthcare costs, morbidity and mortality 62 . Globally as it is recommended in the conference report (2017) of "Action on Antibiotic Resistance (ReAct) Africa Annual Conference, stop or slow the AMR crisis required several and combined actions that include: Frameworks, Raising Awareness, Surveillance, Antimicrobial Stewardship, Infection, Prevention, and Control, Monitoring and Evaluation, inventory and mobilization of resources, as well as strengthen one Health approach, national and international collaboration ${ }^{75}$.

\section{CONCLUSION}

Paucity and/or poor data quality are a chronic problem for the flight of MDROs and NIs. Hence timely and accurate data for MDRB in hospital environment are essential for monitoring the spread of those crises and implementing preventive measures. MDRB are present in a significant level in hospital environment small to large, with or without currently perceived problems with antibiotic resistance. The present study highlights the high contamination of frequently touched Hospital surfaces and frequently used medical device by a variety of nosocomial bacteria associated to high distribution of resistant pattern, to commonly used antibiotics in humans, in Littoral region of Cameroon. MDRB occurred to be a current public health problem. All prevalent bacteria isolated were resistance to more than one drug selected in different or similar groups based on their target site and mode of action. Regrettably, in recognition of these burdens, it is essential that all health facilities periodically and carefully monitor and review emerging AMR patterns so as to prioritize adequate strategies, effective and realistic implementation of health facility level policies and guidelines. Also it is evident that dissemination of antibiotic-resistant pathogens in hospital environment can share with both outpatient setting and animals ecosystem. Therefore, fight against AMR is a global health treat were all hospital personnel, zoologist, botanist and community have their great responsibility. Otherwise, we think it is necessary to pursue this work through studies that can help to understand the reason of the persistence of those various MDRB in various hospital surface by: verifying the contamination of health care givers by those microorganism, evaluating their knowledge and attitude on the infection prevention control and effective use of recommended disinfectant solution on hospital germs in the reduction and elimination of AMR bacteria and NI.

\section{CONTRIBUTIONS OF THE AUTHORS}

MJWT, JCNA, AJ conceived and participated in the design of study. JMWT collected, conducted laboratory assay, analyzed the data and drafted the primary manuscript. JCNA and AJ do interpretation and manuscript design. GOE, XFMK and MNN carried out field activities. All authors contributed to the review and approved the final version.

\section{CONFLICTS OF INTEREST}

All the authors do not have any competing interest.

\section{ACKNOWLEDGEMENTS}

As a PhD part work, we thank all our supervisors. We also thank all the directors, the healthcare workers, and specially the staff of medical laboratory unit of HJMN, MHD, NBDH and MHCN for their cooperation. We also acknowledge Ako simon, Kenfack Lionel, Meyeme Y. Landry, Tegene john, Mamno Jeanne, Manto nayelle, Demguia A. Laure, Bessomo E. Ornella and Gouegni Jipap EPS Takemegni Merline Flaure for their support. We can't end without thanking the academic staff of Faculty of health Sciences of University of Buea especialy Dr Denis Zofou for his orientation.

\section{REFERENCES}

1. Clotilde N, Dieudonné A, André B, Noel A, Gérald S, Basile K, et al. Bacterial ecology of nosocomial infection in intensive care unit of Laquintinie hospital Douala, Cameroon. The Pan African Medical Journal 2013; 14:140.

2. Hortense GK, Etienne G, Michel T, Valantine NN, Calixte DM, Norbert DT et al. Bacteriological assessment of the hospital environment in two referral hospitals in Yaoundé-Cameroon. Pan African Medical Journal 2015; 20:224.

3. Julia L, Vilankar K, Kang H, Brown DE, Mathers A, Barnes LE. Environmental Reservoirs of Nosocomial Infection: Imputation Methods for Linking Clinical and Environmental Microbiological Data to Understand Infection Transmission. AMIA Annu Symp Proc 2017; 2017:1120-9.

4. Smith, J, Adams CE, King MF, Robertson C, Noakes C, Dancer SJ, Is there a relationship between airborne and surface microbes in the critical care environment. J. Hosp. Infect 2018; 100:e123e129.

5. Kouchak F, Askarian M. Nosocomial Infections: The Definition Criteria. Iran J Med Sci 2012; 37(2):72-73.

6. Irene 0, Daniel NT. Knowledge and attitude of healthcare workers and patients on healthcare associated infections in a regional hospital in Ghana. Asian Pac J Trop Dis 2014; 4(2):135-139

7. Heaven S, NEWSLETER healthline ; What is nosocomial infection , Medically Reviewed by Graham Rogers, MD on 24 October 2016 .Also Available : http://www.healthline.com/health/medical board. Accessed on 13 December 2017.

8. Ekanga NG, Hortense GK, Albert SE, William B, Godswill NN, Arthur E, et al. Recommendations on the Fight against Nosocomial Infections Compliance In Health Facilities of Yaounde (Cameroon). IJSRM 2019; 7(8):244-255.

9. Adams CE, Dancer S J. Dynamic Transmission of Staphylococcus aureus in the Intensive Care Unit. Int. J. Environ. Res. Public Health 2020; 17: 2109.

10. Soltani J, Poorabbas B, Miri N, Mardaneh J. Health care associated infections, antibiotic resistance and clinical outcome: A surveillance study from Sanandaj, Iran. World J Clin Cases 2016; 
4(3):63-70.

11. Sievert DM, Ricks P, Edwards JR, Schneider A, Jean P, Srinivasan A Et al. Antimicrobial-resistant pathogens associated with healthcare-associated infections summary of data reported to the national healthcare safety network at the Centers for Disease Control and Prevention, 2009-2010. Infect Control Hosp Epidemiol 2013; 34(1):1-14.

12. Dabar G, Harmouche C, Salameh P, Jaber BL, Jamaleddine G, Waked M Et al. Community-and healthcare-associated infections in critically ill patients: a multicenter cohort study. International Journal of Infectious Diseases 2015; 37:80-85.

13. Kadkhodaei M, Sharif MR, Saba MA, Mousavi GA. Assessing the nosocomial infections' rate and the antibiotic resistance pattern among the patient hospitalized in beheshti hospital during 2013. Int Arch Health Sci 2018; 5:11-5.

14. WHO Healthcare-Associated infections, fact scheet, 2014, WHO, The Burden of Healthcare-Associated infections worldwide: Summary, 2010, and CDC, Vital Signs Report, March 2016.

15. Risk of drug resistance in repeat gram-negative infections among patients with multiple hospitalizations. Available From https://www.sciencedirect.com/science/article/abs/pii/S0883 944117308365. Accessed 22 July 2020.

16. Feleke T, Eshetie S, Dagnew M, Endris M, Abebe W, Tiruneh M, et al. Multidrug-resistant bacterial isolates from patients suspected of nosocomial infections at the University of Gondar Comprehensive Specialized Hospital, Northwest Ethiopia. BMC Res Notes 2018; 11(1):602.

17. Makanjuola OB, Fayemiwo SA, Okesola AO, Gbaja A, Ogunleye VA, Kehinde AO et al. Pattern of multidrug resistant bacteria associated with intensive care unit infections in Ibadan, Nigeria. Ann Ibd. Pg. Med 2018; 16(2):162-169

18. Review on Antimicrobial Resistance. Antimicrobial Resistance: Tackling a Crisis for the Health and Wealth of Nations. 2014.

19. Donkor SE, Codjoe FS. Methicillin Resistant Staphylococcus aureus and Extended Spectrum Beta-lactamase Producing Enterobacteriaceae: A Therapeutic Challenge in the 21stCentury. The Open Microbiology Journal 2019; 13:94-100.

20. CDC. Antibiotic Resistance Threats in the United States, 2019. Atlanta, GA: U.S. Department of Health and Human Services, CDC; 2019.

21. Garba I, Farouq MD, Salisu A, Salisu I, Sirajo HT, Abdulwasiu BT et al.. Laboratory-confirmed hospital-acquired infections: An analysis of a hospital's surveillance data in Nigeria. Heliyon2018; 4: e00720. https://doi:10.1016/j.heliyon.2018.e0072.

22. Citation: McEwen S, Collignon P. Antimicrobial Resistance: a One Health Perspective. Microbiol Spectrum 2018; 6(2): ARBA-00092017.doi: 10.1128/microbiolspec.ARBA-0009-2017.

23. White A, Hughes MJ. Critical Importance of a One Health Approach to Antimicrobial Resistance. EcoHealth 2019; 16:404409. https://doi.org/10.1007/s10393-019-01415-5.

24. Olutola OT, Adeyanju A, Torimiro N. Extended Spectrum $\beta$ lactamase Detection and Antibiotic Susceptibility Profile of Staphylococcus aureus Strains Isolated from Surgical Wound. British Microbiology Research Journal 2016; 17(5):1-7. https://doi.org/10.9734/BMRJ/2016/27848.

25. Ministère de la santé publique. Guide de Bonnes Pratiques d'hygiène en milieu hospitalier. Yaoundé 2017.

26. Cheesbrough M. District laboratory practice in tropical countries. New York: Cambridge University Press; 2006.

27. Clinical and Laboratory Standards Institute (CLSI), Performance Standards for Antimicrobial Susceptibility Testing, Vol. 35, CLSI, Wayne, PA, USA, 2017.

28. Okalla-Ebongue C, Nkodo MR, Nda-Mefo JP, Temfack E, Mengue ER, Adiogo D. Phenotypic Detection of Extended Spectrum $\beta$ Lactamase and AmpC producing Enterobacteriaceae Isolated in A General Hospital. J Microbiol Infect Dis2018; 8(3):113-119.

29. Laboratory detection and reporting of bacteria with extended spectrum $\beta$ Lactamases. Standard Unit, Evaluation and standard laboratory2019; (2) 2: 1-13.

30. CDC ESBL 508. Available from https://www.cdc.gov/drugresistance/pdf/threats-report/esbl508.pdf .Accessed on 22 Jul. 2020.

31. Dancer SJ. Importance of the environment in MRSA acquisition: The case for hospital cleaning. Lancet Infect.Dis 2008; 8: 101113. [CrossRef]

32. Coll F, Harrison EM, Toleman MS, Reuter S, Raven E, Blane B.
Longitudinal genomic surveillance of MRSA in the UK reveals transmission patterns in hospitals and the community. Sci. Transl. Med2017; 9:413. [CrossRef]

33. Hoseini Z A, Shojapour M, Nazari R, Akbari M, Sofian M, Abtahi H. Genotyping of vancomycin resistant enterococci in arak hospitals. Jundishapur J Microbiol 2015; 8(4):e16287.

34. Ferede ZT, Tullu KD, Derese SG, Yeshanew AG. Prevalence and antimicrobial susceptibility pattern of Enterococcus speciesisolated from different clinical samples at Black Lion Specialized Teaching Hospital, Addis Ababa, Ethiopia. BMC Res Notes 2018; 11(1):793.

35. Feleke T, Eshetie S, Dagnew M, Endris M, Abebe W, Tiruneh M, et al. Multidrug-resistant bacterial isolates from patients suspected of nosocomial infections at the University of Gondar Comprehensive Specialized Hospital, Northwest Ethiopia. BMC Res Notes 2018; 11(1):602.

36. Cornejo-Juarez P, Vilar-Compte D , Pe'rez-Jime'nez C, Naamendys-Silva SA, S, Sandoval-Hernandez S, VolkowFernandez $P$.The impact of hospital-acquired infections with multidrug-resistant bacteria in an oncology intensive care unit. International Journal of Infectious Diseases 2015; 31: 31-34

37. Magiorakos AP, Srinivasan A, Carey RB, et al. Multidrugresistant, extensively drug-resistant and pandrug-resistant bacteria: an international expert proposal for interim standard definitions for acquired resistance. Clin Microbiol Infect2012; 18(3):268-281.

38. Ebimieowei E, Ibemologi A. Antibiotics: Classification and mechanisms of action with emphasis on molecular perspectives. Int. J. Appl. Microbiol. Biotechnol. Res2016; 4:90-101.

39. Ye L, Zhenyu G, Ye L, Guoqing H, Ran C, Zhiping C. Impact of nosocomial infections surveillance on nosocomial infection rates : A systematic review. International Journal of Surgery2017; 42:164-169. http://dx.doi.org/10.1016/j.ijsu.2017.04.065.

40. Chun'ai T, Yongxin G, Weidong S, Zhutian L, Xiaolan T. Effectiveness of hospital disinfection and experience learnt from 11 years of surveillance. J Biomed Res 2019; 33:408-413. https://doi.org/10.7555/JBR.33.20180118.

41. Mohamed MMM, Frédéric M, Jene-Francis TKA, Ndode HON , Nabilah PM, Valentine NN et al. Antimicrobial resistance from a one health perspective in Cameroon: a systematic review and meta-analysis. BMC Public Health 2019; 19:1135. https://doi.org/10.1186/s12889-019-7450-5.

42. CDC, the AMR challenge, 2019. Available from : https://www.cdc.gov/drugresistance/intl-activities/amrchallenge.html. Accessed 31/07/20.

43. Costa DM, Johani K, Melo DS, Lopes LKO, Lima LLKO, Tipple AFV et al.. Biofilm contamination of high-touched surfaces in intensive care units: epidemiology and potential impacts. Letters in Applied Microbiolog 2019; 68:269276. https://doi.org/10.1111/lam.13127.

44. Worku T, Derseh D, Kumalo A. Bacterial Profile and Antimicrobial Susceptibility Pattern of the Isolates from Stethoscope, Thermometer, and Inanimate Surfaces of MizanTepi University Teaching Hospital , Southwest Ethiopia. Int J Microbiol 2018; 2018:1-7. https://doi.org/10.1155/2018/9824251.

45. Kwetché FPR, Kougang EG,Noche DC, Youté DN, Tchoukoua HS, Djogang YMA et al. Mitigating hospital infection risks at the "université des montagnes" teaching hospital: holistic policy to Meet the challenge. WJPPS 2020; 9(3):518-535. https://doi.org/10.20959/wjpps20203-15708.

46. Bhatta DR, Hamal D, Shrestha R, Subramanya HS, Baral N, Singh RK, Nayak N and Gokhale S. (2018) Bacterial contamination of frequently touched objects in a tertiary care hospital of Pokhara, Nepal: how safe are our hands? Antimicrob Resist Infect Control. BioMed Central 2018; 7:97. https://doi.org/10.1186/s13756018-0385-2.

47. Aseer et al. Magnitude, diversity, and antibiogram of bacteria isolated from the selected wards of Arba Minch hospital, Arba Minch, Ethiopia 2020. https://doi.org/10.21203/rs.2.23933/v1. Available from https://www.researchsquare.com/article/rs-14494/v1. Accessed 4/08/2020.

48. Ndu IK, Asinobi NI, Ekwochi U, Nduagubam CO, Amadi OF, Okeke $\mathrm{BI}$, Iheji CC, et al.. The bacterial profile and antibiotic sensitivity of the isolated pathogens from medical equipment and surfaces in the children's emergency room of a Nigerian hospital. Medical 
Science and Discovery 2019 6(9):192-7.

http://dx.doi.org/10.36472/msd.v6i9.291.

49. Afle DCF, Alidéhou J A, Roch C J, Olivia H, Sègbè C H, Honoré S B. Healthcare-associated infections: bacteriological characterization of the hospital surfaces in the University Hospital of Abomey-Calavi/so-ava in South Benin (West Africa). BMC Infectious Diseases 2019; 19:28.https://doi.org/10.1186/s12879-018-3648-x.

50. Hearn P, MiliyaT, Seng S, Ngoun C, Day NPJ., Lubell Y, Turner C Et al. Prospective surveillance of healthcare associated infections in a Cambodian pediatric hospital. Antimicrobial Resistance and Infection Control 2017; 6:16. https://doi.org/10.1186/s13756017-0172-5.

51. Saka KH, Akanbi II AA, Obasa TO, Raheem RA, Oshodi AJ, et al. Pathogenic Aerobic Bacterial Contaminants on Non-Critical Hospital Surfaces within Paediatric Ward of a Nigerian Hospital. J Med Microb Diagn 2016; 5:241.

52. Suleyman G, Alangaden G,Bardossy CA.The Role of Environmental Contamination in the Transmissionof Nosocomial Pathogens and Healthcare-Associated Infections. Current Infectious Disease Reports 2018; 20:12.https://doi.org/10.1007/s11908-018-0620-2.

53. Agaba P, Tumukunde P, Tindimwebwa VBJ, Kwizera A. Nosocomial bacterial infections and their antimicrobial susceptibility patterns among patients in Ugandan intensive care units: a cross sectional study. BMC Res Notes 2017; 10:349. https://doi.org/10.1186/s13104-017-2695-5.

54. Nouri F, Karami P, Zarei O, Kosari F, Alikhani YM, Zandkarimi E et al..Prevalence of Common Nosocomial Infections and Evaluation of Antibiotic Resistance Patterns in Patients with Secondary Infections in Hamadan, Iran. Infection and Drug Resistance 2020; 13:2365-2374. https://doi.org/10.2147/IDR.S259252.

55. Gajdács M, Albericio F.Antibiotic Resistance: From the Bench to Patients. Antibiotics 2019; 8: 129 .https://doi.org/doi:10.3390/antibiotics8030129.

56. Atrouni A, Joly-Guillou ML, Hamze M, Kempf M.Reservoirs of Non-baumannii Acinetobacter Species. Front. Microbiol 20167:49. https://doi.org/10.3389/fmicb.2016.00049.

57. Takemegni WJM, Assob NJC, Ateudjieu J, Enow OG, Ngowe NM. (2020) Major Bacteria Species Surface Contaminants in Hospitals of Littoral Region, Cameroon. European Journal of Clinical and Biomedical Sciences 2020; 6(3):26-34.

https://doi.org/10.11648/j.ejcbs.20200603.11.

58. Weber DJ, Rutala WA, Miller MB, Huslage K, Sickbert-Bennett E. Role of hospital surfaces in the transmission of emerging health care-associated pathogens: Norovirus, Clostridium difficile, and Acinetobacter species. Am J Infect Control 2010; 38(5):S25-S33.

59. Hübner NO, Hübner C, Kramer A, Assadian O. Survival of bacterial pathogens on paper and bacterial retrieval from paper to hands: preliminary results. AmJ Nurs 2011; 111(12):30-34

60. Otter JA, Yezli S, Salkeld JA, French GL, Path FRC.Evidence that contaminated surfaces contribute to the transmission of hospital pathogens and an overview of strategies to address contaminated surfaces in hospital settings. Am J Infect Control 2013; 41: S6-S11.

61. Shimose LA, Masuda E, Sfeir M, Berbel Caban A, Bueno MX, dePascale D et al. Carbapenem-resistant Acinetobacter baumannii: concomitant contamination of air and environmental surfaces. Infect Control Hosp Epidemiol 2016; 37(7):P777-781.
62. Canadian Nosocomial Infection Surveillance Program. Healthcare-associated infections andantimicrobial resistance in Canadian acute care hospitals, 2014-2018. Can Commun Dis 2020; 46(5):99-112.https://doi.org/10.14745/ccdr.v46i05a01.

63. ChelliAh A, Ravinder T, Katragadda R, leelA KV, Babu NR. Isolation of MRSA, ESBL and AmpC - $\beta$-lactamases from Neonatal Sepsis at a Tertiary Care Hospital. Journal of Clinical and Diagnostic Research 2014; 8(6):DC24-DC27. https://doi.org/10.7860/JCDR/2014/8597.4512.

64. Chaoui L, RajaaAit M, Fouad M, Naima R. Contamination of the Surfaces of a Health Care Environment by Multidrug-Resistant (MDR) Bacteria. International Journal of Microbiology 2019; 2019:7. https://doi.org/10.1155/2019/3236526.

65. Thean Y T, Jasmine SM T, Huiyi T, Gek H C, Lily SY,Nur S. Multidrug-resistant organisms in a routine ward environment: differential propensity for environmental dissemination and implications for infection control. Journal of Medical Microbiology 2013; 62:766-

772.https://doi.org/10.1099/jmm.0.052860-0.

66. Teghonong J, Cecile OE, Callixte Y, Loick K, Dieudonne A. Phenotypic Characteristics of Klebsiella pneumoniae Extended Spectrum $\beta$-Lactamases Producers Isolated in Hospitals in the Littoral Region, Cameroon. European Journal of Clinical and Biomedical Sciences 2020 6(1):9-

13.https://doi.org /10.11648/j.ejcbs.20200601.13.

67. Rupp ME, Fey PD. ESBL-producing Enterobacteriaceae considerations for diagnosis, prevention and drug treatment. Drugs 2003; 63(4):353-365.

68. Hawkey PM. Molecular epidemiology of clinically significant antibiotic resistance genes. British Journal of Pharmacology 2008; 153: S406-S413.

69. Costerton JW, Stewart PS, Greenberg EP. 1999. Bacterial biofilms: a common cause of persistent infections. Science. 1999; 284:1318- 1322.

70. Mesrati I, Saidani M, Jemili M, Ferjeni S, Slim A, Boubaker IBB. Virulence determinants, biofilm production and antimicrobial susceptibility in Staphylococcus aureus causing deviceassociated infections in a Tunisian hospital. Int J Antimicrob Agents. déc 2018; 52(6):922-9.

https://doi.org/10.1016/j.ijantimicag.2018.05.004.

71. Saxena S, Priyadarshi M, Saxena A, Singh R. Antimicrobial consumption and bacterial resistance pattern in patients admitted in I.C.U at a tertiary care center. J Infect Public Health 2019; 12(5):695-699. https://doi.org/10.1016/j.jiph.2019.03.014.

72. Soltani J, Poorabbas B, Miri N, Mardaneh J. Health care associated infections, antibiotic resistance and clinical outcome: A surveillance study from Sanandaj, Iran. World J Clin Cases 2016; 4(3):63-70. https://doi.org/10.12998/wjcc.v4.i3.63.

73. Agegne M, Abera B, Derbie A, Yismaw G, Shiferaw MB. Magnitude of Vancomycin-Resistant Enterococci (VRE) Colonization among HIV-Infected Patients Attending ART Clinic in West Amhara Government Hospitals. Int J Microbiol 2018; 2018:7. https://doi.org/10.1155/2018/7510157.

74. Murni IK, Duke T, Kinney S, Daley AJ, Soenarto Y. Reducing hospital-acquired infections and improvingthe rational use of antibiotics in a developingcountry: an effectiveness study Arch Dis Child 2014; 0:1-6. https://dx.doi.org/10.1136/archdischild2014-307297.

75. Action on Antibiotic Resistance (ReAct) Africa Annual Conference. (2017) Report. 23. 